\title{
A New Fast Method of Face Authentication based on First Order Statistical Feature
}

\author{
M. Fedias and D. Saigaa \\ Genie electric Department, MSE Laboratory \\ University Mohamed Khider, B.P 145 RP Biskra (07000), Algeria
}

\begin{abstract}
In this paper, we proposed a new fast technique of face authentication based on extraction of a simple Statistical features derived from the image of face. Once the feature vector is extracted, we comparing it with the feature vector of face which is authenticated, and we calculated the error rates in the two sets of validation and test for the data base XM2VTS according to the protocol of Lausanne. The experimental results indicate the reliability, feasibility and efficacy of the proposed method. Moreover, compared to PCA, LDA and EFM, the proposed technique is very quicker to compute.
\end{abstract}

Keywords- statistical pattern recognition, feature extraction, Eigenfaces, Fisherfaces, authentication of face.

\section{INTRODUCTION}

Research in face recognition is currently one of the most important computer vision applications ranging from forensics to surveillance. A successful face recognition methodology depends on the particular choice of the features to represent the face images and the face authentication system knows a priori the identity of the user, the system has to decide whether the a priori user is an impostor or not; it is a binary decision. There are many approaches to facial feature extraction techniques ranging from the Eigenface [1][2] Fisherface [3], independent component analysis ICA [4] and EFM [5] which are a typical dimensionality reduction methods to find a low-dimensional feature space to face recognition. Thus, the execution of LDA or EFM encounters Computational difficulty. Principle Component analysis (PCA) is often used as a pre-processing step to reduce the dimensionality. And LDA is then performed in the lowdimensional PCA subspace where the within-class scatter matrix becomes non-singular. It can be seen that the projection matrices obtained by traditional linear subspace learning methods are related to the statistical characteristics of all training samples. The projection axis tries to preserve (PCA) or discriminate (LDA) all classes. Also, using ICA for face representation it was better than PCA when cosines were used as the similarity measure; however their performance was not significantly different if the Euclidean distance is used. However, ICA; LDA and EFM are both computationally more expensive than PCA [13]. Recently [15] Mohan and al. propose A New Method of Face Recognition based on the second order statistical feature named The texture features witch are derived from co-occurrence parameters with different orientations. But in this paper we propose a new approach using only the first order statistical feature. As a result, the proposed approach has three important advantages over PCA, LDA and EFM. First, it is easier to extract the feature vector directly from the image and we don't need the pre-processing step (PCA) to reduce the dimensionality of all training data. Second, a very less time is required to determine the feature vector from the image of face comparing to the other methods like PCA and LDA because all these methods involves calculating the eigenvectors of a big covariance matrix of all training data. Third, the proposed method didn't need memory than PCA, LDA and EFM [16] which need a memory for the matrix of projection to extract the feature vector. Also, if we need to add one client to our system of authentication ; for our approach is not a problem, we can add it directly to the training data but the difficulty is with the other methods like PCA, LDA and EFM which need to do again all the computations to extract a novel matrix of projection.

The method is simple; the face image is collected by a camera. The subject can arise in front of this one and according to the technique used; the system extracts the features from the face to make the comparison with the features of the claimed person which are preserved in a data base.

Face authentication systems often compare a feature vector $\mathrm{X}$ extracted from the face image to verify with a client template, consisting in similar feature vectors $Y_{i}$ extracted from images of the claimed person stored in a database $(1 \leq \mathrm{i} \leq \mathrm{p}$, where $\mathrm{p}$ is the number of images of this person in the learning set). The matching may be made in different ways, one being to take the Euclidean distance between vectors. If the distance between $\mathrm{X}$ and $\mathrm{Y}_{\mathrm{i}}$ is lower than a threshold, the face from which $\mathrm{X}$ is extracted will be deemed to correspond with the face from which $\mathrm{Y}_{\mathrm{i}}$ is extracted. Choosing the best threshold is an important part of the problem: a too small threshold will lead to a high False Rejection Rate (FRR), while a too high one will lead to a high False Acceptance Rate (FAR); FRR and FAR are defined as the proportion of feature vectors extracted from images in a validation set being wrongly classified, respectively wrongly authentified and wrongly rejected. The validation and test sets must be independent (though with faces of the same people) from the learning set, in order to get objective results. One way of setting the threshold is to choose the one leading to equal FRR and FAR. If the a priori probabilities of having false acceptances (impostors) and false rejections are equal. We use the global threshold leading to FRR $=$ FAR in the remaining of this paper.

This paper is organized as follows: Section II shows how to extract features from images with the proposed method based on Statistical features. Section III shows the experimental results, finally we give the conclusion.

\section{PROPOSED APPROACH}

We are usually interested not in the individual numbers, but rather in certain descriptive quantities such as the average or the variance. In general, the same is true for the image of face. If we consider the face image as a matrix where each row and column represent a collection of numbers described by certain descriptive quantities like mean, standard deviation, skewness, kurtosis...etc. So the feature vector is the combination between these quantities of each row and column of the image. 
The proposed method proceeds as follows:

Let $A=\left(x_{1} x_{2} \ldots x_{i} \ldots x_{N}\right)$ represent the $(n \times N)$ data matrix, where each $x_{i}$ is the feature vector of dimension $n$. Here $\mathrm{n}$ represents the total number of elements in the feature vector and $\mathrm{N}$ is the number of face images in the training set.

The feature vector $x_{i}$ is the combination between the descriptive quantities of each row and column of the image. So we have reduced the size of image from $(r \times c)$ to a feature vector of size $n=(q \times(r+c))$. Where q represents the number of descriptive quantities, $\mathrm{r}$ and $\mathrm{c}$ are the number of rows and columns of the face image.

Afterwards, we apply a photonormalization (eq.6). That wants quite simply to say that for each feature vector, we withdraw from each element the average value of those on the the feature vector, and that we divide those by their standard deviation. Finally we make standardization. The photonormalization acts on one feature vector where as standardization acts on a group of the data matrix (for each component, one withdraws the average of this component for all the feature vectors of the data matrix of training and one divides by the standard deviation).

Here we discuss some descriptive quantities $[9][10][11][12][13][14]$ :

\section{a. Mean}

The arithmetic mean, defined by :

$$
\boldsymbol{\mu}=\frac{\sum_{i=1}^{n} x_{i}}{n}
$$

\section{b. Variance}

The variance is an important quantity defined by :

$$
\operatorname{Var}=\frac{\sum_{i=1}^{n}\left(x_{i}-\mu\right)^{2}}{n}
$$

\section{c. Standard Deviation}

The standard deviation (Std) is defined as the square root of the variance:

$$
\sigma=\sqrt{\frac{\sum_{i=1}^{n}\left(x_{i}-\mu\right)^{2}}{n}}
$$

\section{d. Skewness}

Skewness is the third central moment of $\mathrm{x}$, divided by the cube of its standard deviation.

$$
S=\frac{\sum_{i=1}^{n}\left(x_{i}-\mu\right)^{3}}{n}
$$

\section{e. Kurtosis}

Kurtosis is the fourth central moment of $\mathrm{x}$, divided by fourth power of its standard deviation.

$$
K=\frac{\sum_{i=1}^{n}\left(x_{i}-\mu\right)^{4}}{n}
$$

The photonormalization is defined by :

$$
\operatorname{phot}(x)=\frac{x-\mu_{x}}{\sigma_{x}}
$$

The principal of this system is the extraction of a feature vector of an individual, in order to compare it with a vector Yi which contain the feature of this same individual extracted starting from his images which are stored in a data base.

Also we can use the standard deviation for detecting the components of human face like eyes; mouth and nose which are located at the maxima of the standard deviation and the figure 1 explain this idea clearly.

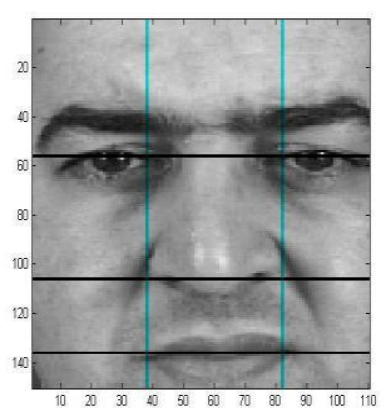

(a)

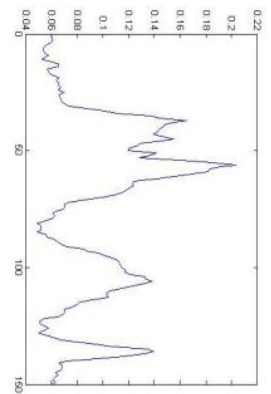

(b)

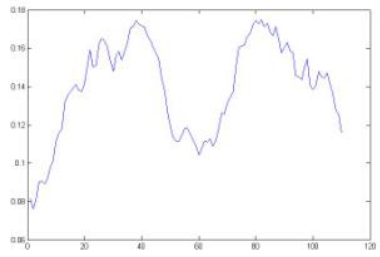

(c)

Fig.1 (a) image of face (b) Vertical Standard deviation (c) Horizontal Standard deviation . Detection of eyes, mouth and nose of a human face by the standard deviation of each row and column of the image.

We observe that this method is very interesting and easy and faster for searching the position of different parts of the face image.

\section{EXPERIMENTAL RESULTS}

\section{III.1. Data base XM2VTS}

Our experiments were carried out on frontal face images of the data base XM2VTS. The principal choice of this data base is its big size, with 295 people and 2360 images in total and its popularity, since it became a standard in the audio and visual biometric community of multimodal checking of identity [7]. For each person eight catches were carried out in four sessions distributed for five months.

The protocol related to XM2VTS divides the base into two categories 200 clients and 95 impostors; the people are of the two sexes and various ages. The photographs are color of high quality and size (256x256). 
The protocol of Lausanne shares the data base in three sets [8]:

1. The set of training: it contains information concerning the known people of the system (only clients)

2. The set of evaluation (validation): allows fixing the parameters of the face authentication system.

3. The set of test: allows to test the system by presenting images of people to him being completely unknown to him.

For the class of impostors, 95 impostors are divided in two sets: 25 for the set of evaluation and 75 for the set of test.

The sizes of the various sets are included in table I.

Table.I

Photos distribution in the various sets

\begin{tabular}{|ccc|}
\hline Set & Clients & Imposters \\
\hline Training & 600 & 0 \\
& (3 by subject) & 600 \\
Evaluation & (3 by subject) & 200 \\
& 400 & (8by subject) \\
Test & (2 by subject) & (8by subject) \\
\hline
\end{tabular}

The next figure represents some examples of faces in the data base XM2VTS, the people are of the two sexes and various ages.

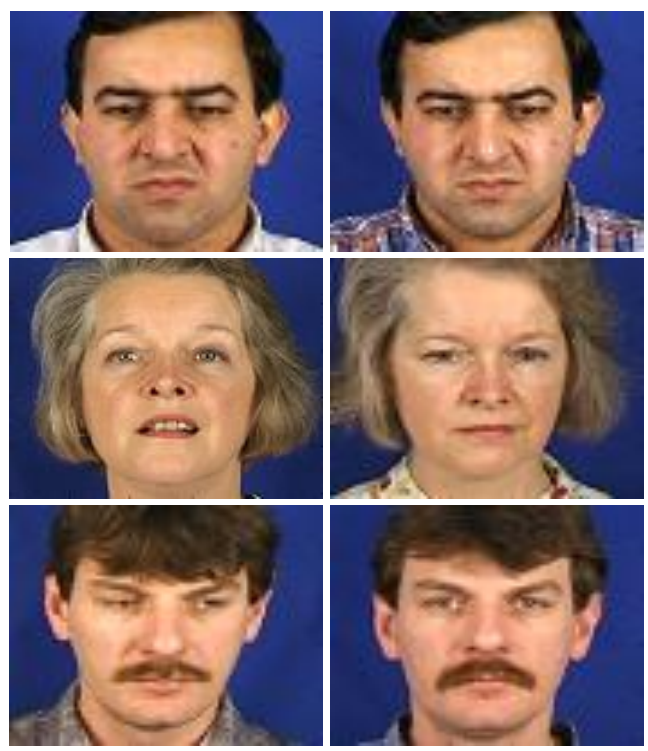

Fig.2 represents some examples of faces images in the data base XM2VTS.

\section{III.2. Pretreatment}

By looking at the images we clearly note the appearance of characteristics not desired on the level of the neck, like the collars of shirt, the sports shirts, etc. In addition, the hair is also a characteristic changing during the time (change of cut, colour, baldness...). The background appears on the images; it is used for nothing, and inflates the size of the data unnecessarily. Finally the ears cause also a problem. Indeed, if the person presents herself slightly differently in front of the camera (rotation), we can see only one ear. This is why we decided to cut the image vertically and horizontally and to keep only one window of size $150 \times 110$ cantered on the face. This window is manually extracted from the frontal image.

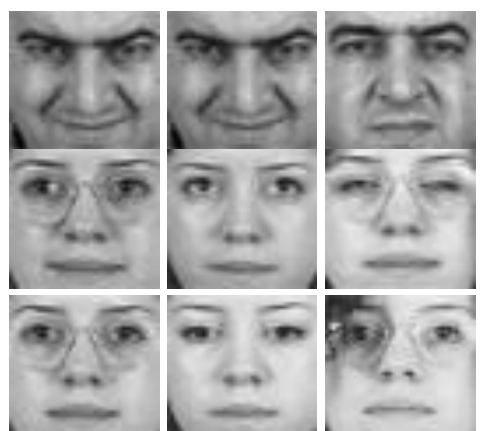

Fig 3. Example XM2VTS images used in our experiments (cropped to the size of $150 \times 110$ to extract the facial region.

\section{III.3. Similarity measures and classification}

The similarity measures used in our experiments to evaluate the efficiency of different representation and authentication method is correlation similarity measure, which are defined as follows:

$$
\operatorname{Corr}(A, B)=\sum_{i=1}^{N} \frac{\left(A_{i}-\mu_{A}\right)\left(B_{i}-\mu_{B}\right)}{\sigma_{A} \sigma_{B}}
$$

The threshold is fixed to have FAR= FRR on evaluation set; finally, the performances of the method (including the threshold value) are measured on an independent test set (on this set, FAR will not be necessarily equal to FRR).

\section{III.4. Results}

In an authentication system of face the goal is a binary decision client or impostor with the rate minimum of equal error in test. The equal error rate FAR=FRR obtained on the validation set and the test set in face authentication performance of this method apply the correlation distance measure is shown in table III, and one can see from the table that the use of the mean alone or the standard deviation with photonormalization and correlation distance gives a rate of success about $85.66 \%$ and $84.06 \%$ respectively. And it has been found experimentally an improvement in the success rate if we combined the different statistical quantities mean and standard deviation, mean and variation, mean and skewness, mean and kurtosis, or all these quantities together; we found these success rates respectively: $89.48 \%, 78.38 \%, 86.96 \%$, $85.50 \%$, and $70.35 \%$.

In particular, the combination of the mean and the standard deviation (MS) gives the best rate of success than the other combinations if we applied a photonormalization and Correlation distance for classification. Where The MS method gives the best rate of success about $89.48 \%$ on the test set. In general, the MS method is faster comparing to the PCA, LDA and EFM because they involves calculating the eigenvectors of a big covariance matrix. Also we don't need any memory with MS method than PCA and LDA who need a memory to preserve the space of projection. But the problem of illumination variation, facial expression, aging, are still exist.

It is also noticed that the error rates in evaluation and test sets are near by using this method that signified then that the proposed system for face authentication is more stable.

The figure 6 represents different distances intra for clients and extra for impostors in the two sets of evaluation and test set using the MS method. 
The next table shows the CPU time to calculate the matrix of projection and the time to extract the feature of one image obtained by different methods, also the success rate of each method using the XM2VTS database.

TABLE II

Comparison of the performance of MS, PCA, LDA and EFM Using XM2VTS Database (Pentium 4, 1.6GHZ).

\begin{tabular}{|c|c|c|c|}
\hline Method & $\begin{array}{c}\text { Success rate SR } \\
(\%)\end{array}$ & $\begin{array}{c}\text { CPU Time } \\
\text { of Matrix } \\
\text { Projection } \\
(\mathrm{s})\end{array}$ & $\begin{array}{c}\text { CPU Time } \\
\text { To extract } \\
\text { feature of } \\
\text { one } \\
\text { image(s) }\end{array}$ \\
\hline MS & 89.48 & 0 & 0.09 \\
PCA & 88.70 & 47.84 & 0.120 \\
LDA & 93.03 & 119.10 & 0.100 \\
EFM & 94.68 & 56.64 & 0.110 \\
\hline
\end{tabular}

Form this table we observe that the MS method is better than PCA but LDA, EFM still more better than PCA and MS method in term of success rate. But in fact our approach MS have many advantages over the other methods, it is simpler easier and faster; it required a very very less time about 0.09 to extract the feature vector by only a two simple quantities the mean and the standard deviation of the image directly. The feature extraction with MS method is more than 500 times faster than PCA and 1000 times faster than LDA. Mainly because the latter involves calculating the eigenvectors of a big covariance matrix. Also, we don't need a PCA as a preprocessing step to reduce the dimensionality of all training data like LDA and EFM.

In order to reduce the dimensionality of the feature vector of the MS method we have applied a wavelet by a filter $9 / 7$ to each image of face, and the figure 4 explain the three stage transform of the wavelet $9 / 7$, and the success rate are shown in the next figure.

Original $(150 * 110) 520$

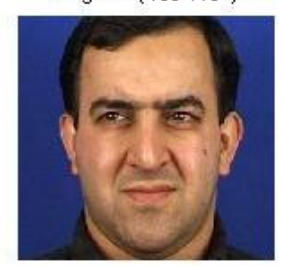

2-stage transform 128

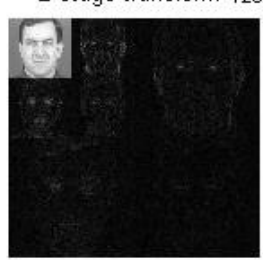

Fig4 the three stages transform of the wavelet $9 / 7$ of the face

image
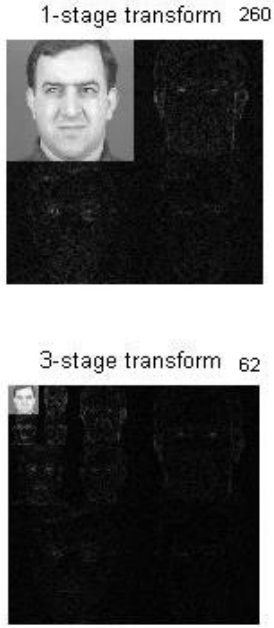

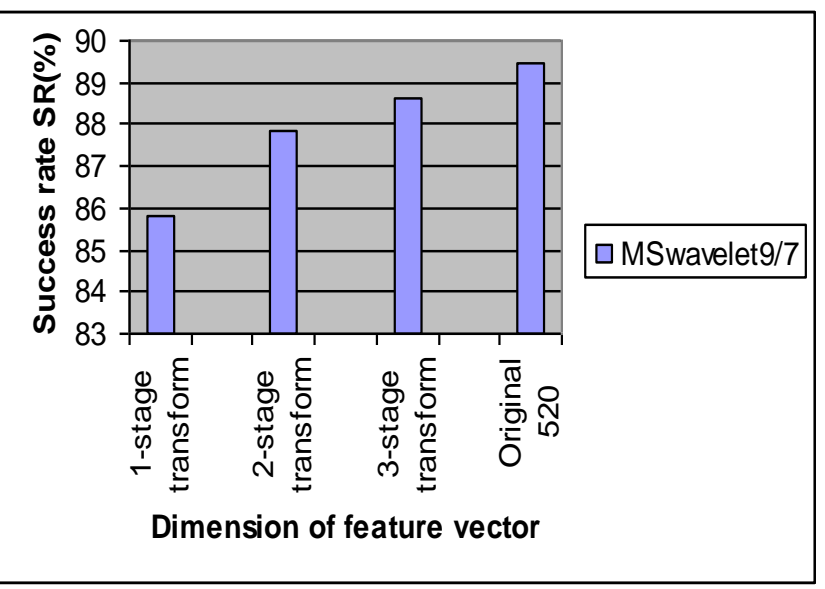

Fig5 Results of the MS method applying the wavelet 9/7

We find that when the feature vector was reduced to 62 with the 3-stage transform by a wavelet $9 / 7$; the success rate is $85.80 \%$ witch is still good enough compared to the success rate with the size of the original feature vector about 520 and also compared to PCA with the same size.

\section{CONCLUSION}

In this paper, a new technique for image feature extraction and representation was developed. Especially with The MS method which has many advantages. In the first place, since MS method based in mean and standard deviation of the image, it is simpler and faster than the PCA, LDA and EFM which involves calculating the eigenvectors of a big Covariance matrix of all training data. Second, it is better to use for authentication systems of face in terms of success rate than PCA. Third, MS method didn't need memory than PCA, LDA or EFM which need a memory for the matrix of projection. Also, if we need to add one client to our system of authentication ; for our approach is not a problem, we can add it directly to the training data but the difficulty is with the other methods like PCA, LDA and EFM which need to do again all the computations to extract a novel matrix of projection. But the difficulty caused by illumination variation, facial expression and aging are still exists.

In further works, we propose the use of color to improve the performance of this system in term of success rate.

\section{REFERENCES}

[1] Turk M. A. et Pentland A. P.: Face recognition using eigenfaces. IEEE Comput. ScoPress, p. 586-591, June 1991.

[2] Matthew Turk et Alex Pentland : Eigenfaces for recognition. Journal of cognitive neuroscience, 3(1):7186, 1991.

[3] Belhumer, P. N., Hespanha, J. P., Kriegman, D. J,(1997) Eigenfaces vs. Fisherfaces: recognition using class specific linear projection, IEEE Trans. Pattern Anal Mach. Intell. 19 (7) 711-720.

[4] M.S. Bartlett, J.R. Movellan, and T.J. Sejnowski ‘ face recognition by independent component anlysis' IEEE Trans. Neural Networks, Vol.13 no. 6, pp 1450$1464,2002$. 
[5] Chengjun Liu and Harry wechsler ' A shape and Texture Based Enhanced Fisher Classifier for face recognition' IEEE Trans. On image processing, Vol, 10, No.4 pp, 598_608, April 2001.

[6] M.H. Yang, ' Kernel Eigenfaces vs. Kernel Ficherfaces: Face recognition using Kernel methods “ Proc. Fifth IEEE int'l conf. Automatic face and gesture recognition (RGR'02) , pp. 215-220, May 2002

[7] K. Messer, J. Matas, J. Kittler et K. Jonsson : $\mathrm{Xm} 2 \mathrm{vtsdb}$ : The extended m2vts database. Audio- and Video-based Biometric Person Authentication (AVBPA), pages 72-77, Mars 1999.

[8] J. Luettin and G. Maitre. "Evaluation protocol for the extended M2VTS database". IDIAP, available at: http://www.ee.surrey.ac.uk/Research/VSSP/xm2vtsdb/ face- avbpa2001/protocol.ps, 1998.

[9] W.J. DeCoursey , Statistics and Probability for Engineering Applications With Microsoft Excel, College of Engineering,University of Saskatchewan,Saskatoon ,Copyright 2003, Elsevier Science (USA).

[10] Fukunaga, K.,(1990). Introduction to Statistical Pattern Recognition, second ed., Academic Press Springer, New York.

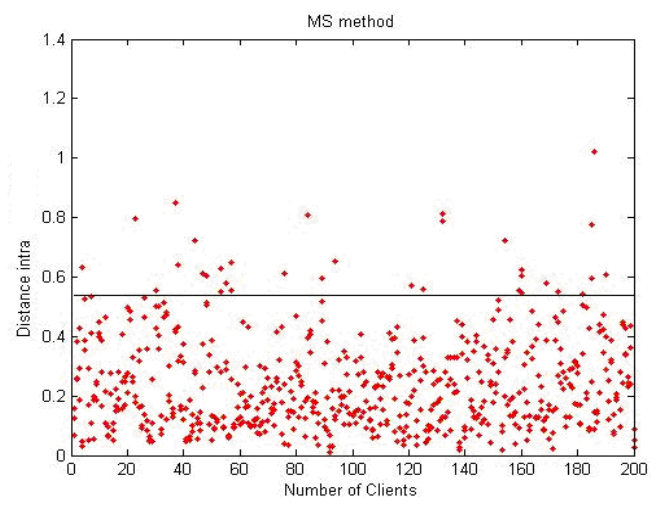

(a)

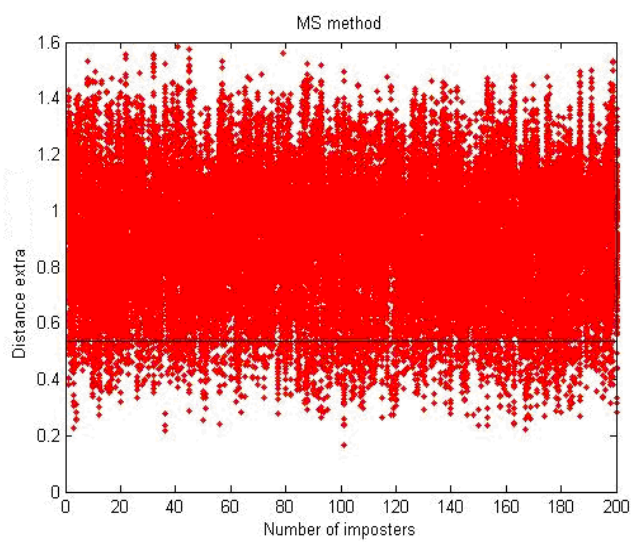

(c)
[11] M. Li and B. Yuan. 2D-LDA: A statistical Linear Discriminant Analysis for image matrix. Pattern Recognition Letters, 26:527-532, 2005.

[12] L.F Chen et Al. Why recognition in a statistics based face recognition system should be based on the pure face purtion : a probabilistic decision based proof Pattern recognition. Vol 34,No 7. 2001.

[13] Delac K., Grgic M., Grgic S., Independent Comparative Study of PCA, ICA, and LDA on the FERET Data Set, International Journal of Imaging Systems and Technology, Vol. 15, Issue 5, 2006, pp. 252-260.

[14] J. Short, J. Kittler and K. Messer, "A Comparison of Photometric Normalisation Algorithms for Face Verification" IEEE Conf. Automatic Face and Gesture Recognition 2004,

[15] Mohan et al. 'A New Method of Face Recognition Based on Texture Feature extraction on individual components of face / pp. 69-74 International Journal of Signal and Image Processing (Vol.1-2010/Iss.2).

[16] M.Fedias, D.Saigaa' Nonlinear fusion of colors to face authentication using EFM method' Journal of applied computer science \& mathematics, no. 9 (4) /2010, Suceava, Romania.

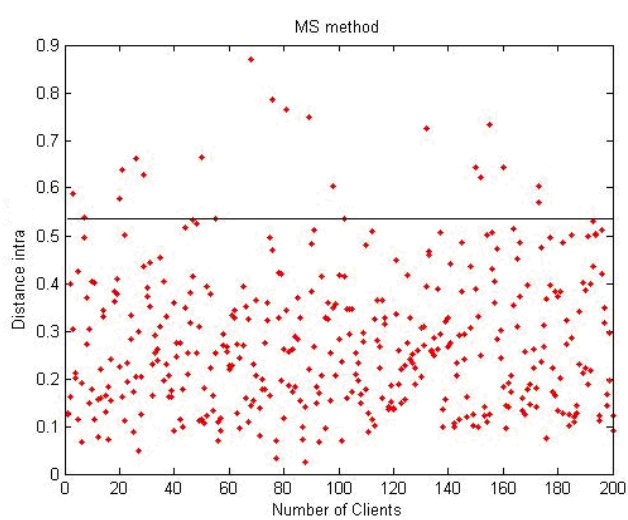

(b)

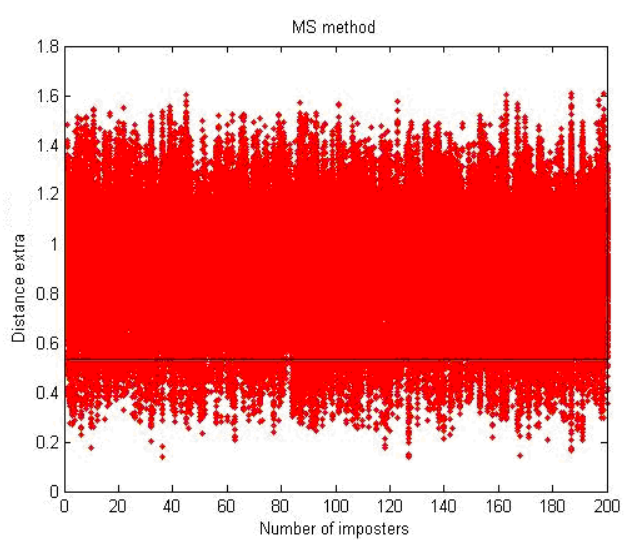

(d)

Fig.6 Different distances by MS method. (a) Distance intra by MS of the evaluation set (b) distance intra by MS of the test set (c) Distance extra by MS of the evaluation set (d) distance extra by MS of the test set. 
TABLE III

Different errors rate of the proposed method

\begin{tabular}{|c|c|c|c|c|c|c|}
\hline \multirow{2}{*}{$\begin{array}{l}\text { Type of distance } \\
\text { 'Correlation' }\end{array}$} & \multirow{2}{*}{$\begin{array}{c}\begin{array}{c}\text { Validation } \\
\text { Set }\end{array} \\
\text { FAR }= \\
\text { FRR }\end{array}$} & \multicolumn{4}{|c|}{ Test Set } & \multirow{2}{*}{$\begin{array}{l}\text { Dimension of } \\
\text { feature vector }\end{array}$} \\
\hline & & FAR & FRR & $(\mathrm{FAR}+\mathrm{FRR}) / 2$ & $\begin{array}{c}\text { Success rate SR } \\
(\%)\end{array}$ & \\
\hline Mean & 6.99 & 7.59 & 6.75 & 7.17 & 85.66 & 260 \\
\hline Std & 7.04 & 8.94 & 7.00 & 7.97 & 84.06 & 260 \\
\hline Skewness & 7.81 & 8.09 & 9.75 & 8.92 & 82.16 & 260 \\
\hline Kurtosis & 10.3 & 10.32 & 14.00 & 12.16 & 75.68 & 260 \\
\hline Variance & 8.14 & 9.87 & 8.00 & 8.93 & 82.14 & 260 \\
\hline MS & 5.47 & 5.77 & 4.75 & 5.26 & 89.48 & 520 \\
\hline Mean and Var & 10.03 & 9.37 & 12.25 & 10.81 & 78.38 & 520 \\
\hline Mean and & 5.67 & 5.05 & 8.00 & 6.52 & & 520 \\
\hline Skewness & & & & & 86.96 & \\
\hline Mean and & 5.51 & 5.75 & 8.75 & 7.25 & & 520 \\
\hline Kurtosis & & & & & 85.50 & \\
\hline Mean Var & & & & & & \\
\hline $\begin{array}{c}\text { Skewness and } \\
\text { Kurtosis }\end{array}$ & 12.69 & 13.90 & 15.75 & 14.80 & 70.35 & 1040 \\
\hline
\end{tabular}

